\title{
Tumor neuroendocrino ano-rectal: desde un pólipo con buen pronóstico hasta un carcinoma letal
}

\author{
Ano-rectal neuroendocrine tumour: from a polyp with a good prognosis to a lethal \\ carcinoma
}

\author{
Beatriz De Andrés-Asenjo ${ }^{1 *}$, Francisco J. Ortiz de Solórzano-Aurusa ${ }^{1}$, Henar Borrego-Pintado², Francisco \\ Blanco-Antona ${ }^{1}$, Alejandro Romero-de Diego y Juan Beltrán de Heredia-Rentería ${ }^{1}$ \\ ${ }^{1}$ Servicio de Cirugía General y Aparato Digestivo; ${ }^{2}$ Servicio de Anatomía Patológica. Hospital Clínico Universitario de Valladolid, Valladolid, España
}

\begin{abstract}
Resumen
Introducción: Aunque los tumores neuroendocrinos rectales son neoplasias poco frecuentes, su incidencia está aumentando en las tres últimas décadas. El objetivo de este trabajo es el estudio del comportamiento de dichos tumores basándonos en sus variables clínicas, diagnósticas, terapéuticas y anatomopatológicas en un hospital terciario. Método: Estudio retrospectivo de los tumores neuroendocrinos rectales desde el año 2000 hasta el año 2017 en nuestro hospital. Se realizó una búsqueda informatizada en el programa SNOMED del servicio de anatomía patológica, empleando los criterios «tumor neuroendocrino" $y$ «tumor carcinoide». Resultados: Se hallaron 16 pacientes con tumor neuroendocrino ano-rectal. El 75\% eran varones. La mediana de edad fue de 53 años (rango: 34-83). El diagnóstico fue incidental en el 62,5\%. El tratamiento fue endoscópico en 13 (81\%) pacientes y quirúrgico en 3 (19\%). El 75\% de las lesiones fueron T1. El 68,7\% de los tumores presentaban un grado de agresividad G1. La media de seguimiento fue de 29 meses; no hubo recidivas. Durante el seguimiento fallecieron $3(18,5 \%)$ pacientes, todos ellos con tumor G3 metastásico, y la supervivencia media fue de 8 meses. Conclusiones: Los tumores neuroendocrinos rectales son cada vez más frecuentes y presentan unos patrones variables, cuyo pronóstico dependerá de su agresividad histológica. El aumento de su incidencia puede deberse a una mayor concienciación de la existencia de estos tumores, a las campañas de cribado del cáncer colorrectal, al aumento del número de colonoscopias realizadas y a la generalización de los estudios histológicos con marcadores neuroendocrinos.
\end{abstract}

PALABRAS CLAVE: Carcinoide rectal. Tumor neuroendocrino. Neoplasia rectal.

\begin{abstract}
Background: Although rectal neuroendocrine tumours are rare neoplasms, their incidence is increasing in the last three decades. The objective of this work is to study the behaviour of these tumours based on their clinical, diagnostic, therapeutic, and pathological variables in a tertiary hospital. Method: Retrospective study of rectal neuroendocrine tumours from 2000 to 2017 in our hospital. A computerized search was performed in the SNOMED program of the pathological anatomy service, using the criteria "neuroendocrine tumour" and "carcinoid tumour". Results: We found 16 patients with ano-rectal neuroendocrine tumour; 75\% were male. The median age was 53 years (range: 34-83). The diagnosis was incidental in 62.5\%, with endoscopic treatment in 13 patients (81\%) and surgical treatment in $3(19 \%) .75 \%$ of the lesions were $11.68 .7 \%$ of the tumours showed a degree of G1 aggression. The mean follow-up was 29 months; no recurrences. During follow-up, three patients died (18.5\%), all of them with metastatic G3 tumour and the mean survival was 8 months. Conclusions: The rectal neuroendocrine tumours are increasingly frequent and present variable patterns, whose prognosis will depend on their histological aggressiveness. The increase incidence may be due to a greater awareness of the existence of these tumours, to colorectal cancer
\end{abstract}

\section{Correspondencia:}

*Beatriz de Andrés-Asenjo

Ramón y Cajal, s/n

47005 Valladolid, España

Fecha de recepción: 25-03-2018

Fecha de aceptación: 24-04-2018

E-mail: beatrizdeandres007@yahoo.es

DOI: $10.24875 / C I R U .18000302$

Cir Cir. 2018;86:515-521

Contents available at PubMed www.cirugiaycirujanos.com 
screening, to the increase in the number of colonoscopies performed and to the generalization of histological studies with neuroendocrine markers.

KEY WORDS: Rectal carcinoid. Neuroendocrine tumour. Rectal neoplasm.

\section{Introducción}

En el año 1888 Lubarsch describió un tipo de tumores originados en las células neuroendocrinas ${ }^{1}$, y en 1907 Obendorfer denominó a estos tumores «carcinoides» (tumor parecido a un carcinoma) ${ }^{2}$; en la actualidad, se recomienda emplear el término «tumor neuroendocrino» (TNE) por su mayor precisión al describir su etiología y localización ${ }^{3-6}$.

Los TNE rectales (TNER) suponen el $17.7 \%$ del total de los TNE y el $27 \%$ de los tumores gastro-entero-pancreáticos (TNEGEP). Su incidencia aproximada es de 0.86 por 100,000 habitantes, con un incremento anual del $8.2 \% \%^{7,8}$. En los EE.UU. son los TNE más frecuentes, sobre todo en varones de raza negra, y parece existir una mayor predisposición en la población asiática ${ }^{5,8-12}$. En el Registro Español de Tumores Neuroendocrinos (RGETNE) ocupan el cuarto lugar (6\%) de los TNEGEP, tras los tumores de intestino delgado, apéndice y estómago ${ }^{13}$.

Los TNER son generalmente tumoraciones pequeñas y únicas, que se diagnostican con mayor frecuencia en pacientes asintomáticos durante una colonoscopia realizada en una campaña de detección precoz del cáncer o para el estudio de otra patología colorrectal ${ }^{12}$. En ocasiones presentan síntomas locales, como sangrado rectal, dolor o alteraciones del ritmo intestinal, siendo el síndrome carcinoide de aparición infrecuente. Su tratamiento varía desde la polipectomía endoscópica hasta la escisión transanal, por lo que tanto este como el pronóstico van a depender del tamaño tumoral y de la invasión de la pared rectal, ${ }^{5,12,14-16}$.

El objetivo de este trabajo es una revisión de los TNER diagnosticados en un hospital terciario durante 16 años, basándonos en sus aspectos clínicos, diagnósticos, terapéuticos, pronósticos y anatomopatológicos.

\section{Método}

Se realiza un estudio retrospectivo de los pacientes diagnosticados de TNE ano-rectal entre enero del año 2000 y enero del año 2017. El estudio fue aprobado por el comité ético de nuestro hospital, forma parte del proyecto de investigación Estudio de los tumores neuroendocrinos gastrointestinales y pancreáticos, aprobado por la Comisión de Investigación del Hospital Clínico Universitario de Valladolid (España), y ha sido realizado tras obtener el consentimiento informado por escrito de todos los pacientes después de haberles explicado el procedimiento.

Para la búsqueda informatizada se emplearon los criterios «tumor neuroendocrino" y "carcinoide» en el programa SNOMED del servicio de anatomía patológica.

Se estudiaron las siguientes variables: edad, sexo, manifestaciones clínicas, antecedentes de patología colorrectal, localización del tumor, marcadores en sangre de cromogranina $\mathrm{A}(\mathrm{CgA})$ y en orina de 5-hidroxiindolacético (5-HIAA), presencia de metástasis, tipo de tratamiento (endoscópico o quirúrgico), complicaciones posquirúrgicas, mortalidad, necesidad de tratamiento adyuvante, tiempo de seguimiento, clasificación TNM, estadio y grado de agresividad tumoral.

En todos los casos se realizaron, además de la tinción de hematoxilina-eosina, técnicas de inmunohistoquímica para los marcadores neuroendocrinos $\mathrm{CgA}$ y sinaptofisina, así como el índice de proliferación Ki-67 (Fig. 1).

Para la clasificación y la estadificación de los tumores se empleó el sistema TNM de la Unión Internacional Contra el Cáncer del año $2009^{17}$,y para la gradación de la agresividad se utilizaron los criterios de la Organización Mundial de la Salud del año 2010 para los TNEGP ${ }^{18}$.

La base de datos y su análisis descriptivo se realizaron con el paquete estadístico IBM SPSS versión 20.

\section{Resultados}

Se diagnosticaron 16 TNER en los 17 años revisados, 12 en varones (75\%) y $4(25 \%)$ en mujeres. El $81.3 \%(n=13)$ de los tumores fueron diagnosticados en los últimos 4,5 años del estudio (Fig. 2). La media de edad fue de 58 años, con una mediana de 53 (rango: 34-83).

El $62.5 \%(n=10)$ de los TNER se presentaron en pacientes asintomáticos, siete en las colonoscopias realizadas durante la campaña de cribado del cáncer colorrectal y los otros tres durante el control de una colitis ulcerosa, en el estudio de una hemorragia digestiva alta y durante el diagnóstico de un cuadro de estreñimiento crónico. Entre los pacientes sintomáticos, cinco presentaron rectorragia y otro una 


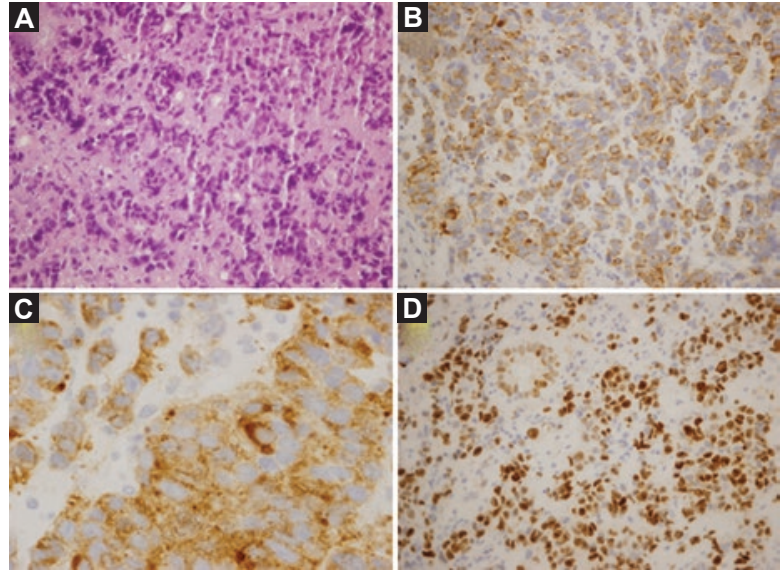

Figura 1. Estudio histológico de los tumores neuroendocrinos rectales. A: hematoxilina-eosina 40x. B: cromogranina positiva 40x. C: sinaptofisina positiva 40x. D: Ki-67 > 20\%.

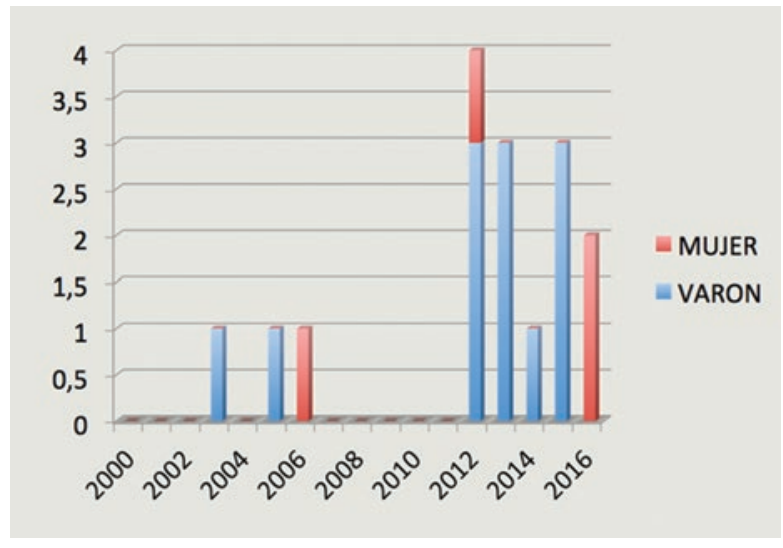

Figura 2. Incidencia por año y sexo de 16 tumores neuroendocrinos rectales.

tumoración dolorosa en el canal anal. En el momento del diagnóstico, ningún paciente presentaba un síndrome carcinoide típico con flush cutáneo y diarrea, aunque sí se objetivó este síndrome durante el seguimiento de dos tumores con diseminación a distancia.

En $5(31 \%)$ pacientes existían antecedentes de patología colorrectal: tres pólipos, una colitis ulcerosa y una neoplasia de recto tratada con resección endoanal.

El diagnóstico de TNER se estableció tras la realización de colonoscopia y biopsia de la tumoración. Todos fueron lesiones únicas y su localización más frecuente fueron los dos tercios inferiores del recto (14 casos, $87,5 \%$ ), excepto un tumor que se encontraba en el tercio superior rectal y otro en el canal anal (Fig. 3).

Las concentraciones séricas de $\mathrm{CgA}$ se analizaron en $9(56 \%)$ casos, y se encontraron elevadas en $3(33 \%)$. El 5-HIAA en orina se estudió en $10(62,5 \%)$ casos, y fue normal en el $100 \%$.

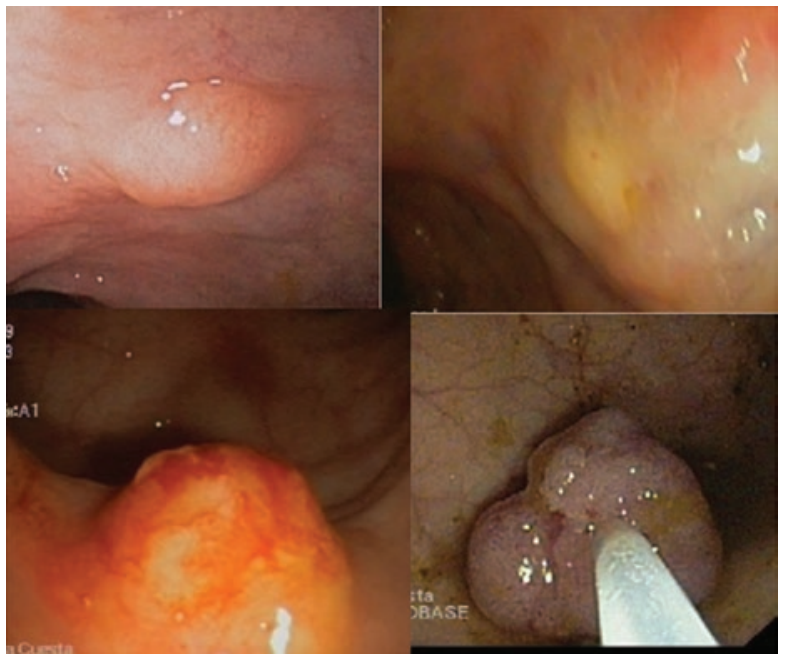

Figura 3. Aspectos endoscópicos de los tumores neuroendocrinos rectales.

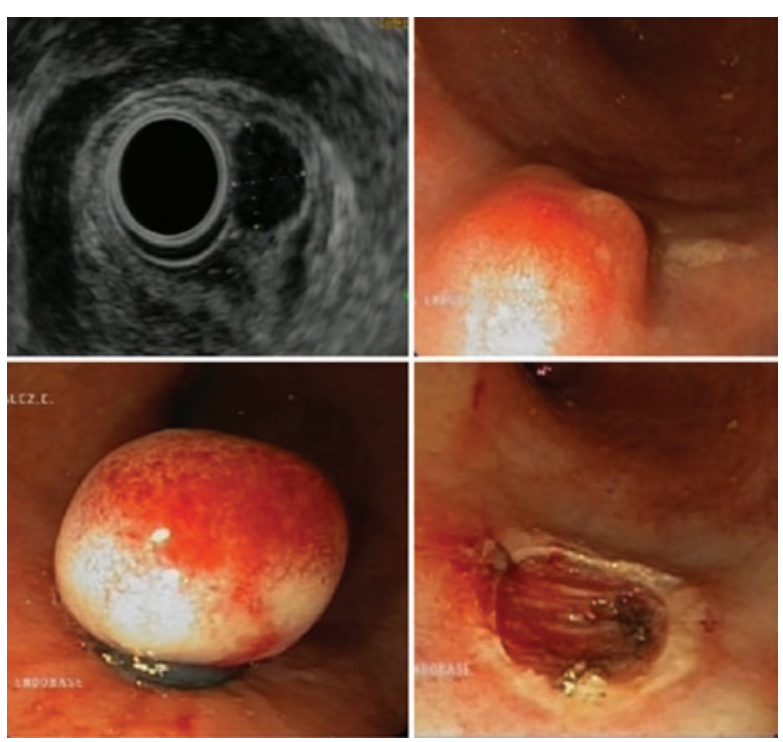

Figura 4. Tumor neuroendocrino rectal: ecoendoscopia (imagen izquierda arriba) y polipectomía con banda elástica.

Como estudios complementarios para el diagnóstico y el seguimiento se emplearon:

- Tomografía computarizada (TC) en 14 (87,5\%) pacientes, que fue patológica en $5(35,7 \%)$ al detectar metástasis hepáticas, óseas y diseminación ganglionar regional. Como hallazgo incidental, en un paciente se diagnosticó un mucocele apendicular.

- Gammagrafía con análogos de la somatostatina (GASS) en 10 (62,5\%) pacientes; en nueve de ellos se asoció a una TC por emisión de fotón único (SPECT). En el momento del diagnóstico fue normal en el $100 \%$ de los casos, y durante el seguimiento de un tumor G3 se detectó una metástasis hepática. 
- Ultrasonografía endoscópica (USE) en 8 (50\%) pacientes, de los cuales en dos se realizó previamente a la resección tumoral y mostró la existencia de una tumoración sin invasión de la muscular propia ni afectación ganglionar (Fig. 4). En los seis pacientes restantes se empleó durante el seguimiento, sin hallar ninguna lesión.

- Se realizaron cinco resonancias magnéticas (RM), en las que se diagnosticaron dos diseminaciones ganglionares y una metástasis ósea.

- En un tumor indiferenciado de canal anal, con GASS negativa, se realizó una tomografía por emisión de positrones (PET) en la que se evidenció diseminación ganglionar regional.

Se realizó tratamiento endoscópico a 13 (81\%) pacientes: 10 resecciones simples y una con ligadura elástica (Fig. 4); una tumoración estenosante con diseminación metastásica se trató con una endoprótesis colónica, y en un tumor metastásico se realizó biopsia.

Tres (18,7\%) pacientes fueron intervenidos quirúrgicamente. Las técnicas empleadas fueron una exéresis de la tumoración anal y biopsia ganglionar inguinal, una resección endoanal y una amputación abdominoperineal. No hubo mortalidad perioperatoria. La amputación abdominoperineal cursó con infección de la herida perineal. En el caso del mucocele apendicular asociado al TNER, se practicó una apendicectomía laparoscópica programada.

La clasificación TNM, el estadio y el grado de agresividad en el momento del diagnóstico se muestran en la tabla 1. El $75 \%(n=12)$ de las lesiones fueron T1 y el $69 \%(n=11)$ estaban en estadio I y G1. Todos los pacientes con un tumor $\mathrm{G} 3(\mathrm{n}=5)$ presentaban afectación ganglionar regional, y tres de ellos tenían metástasis a distancia.

El informe anatomopatológico no especificaba el estado de los bordes de resección en $7(43,7 \%)$ casos, en $3(18,7 \%)$ estaban libres y en $6(37,5 \%)$ se encontraban afectados. Dos tumores $\mathrm{G} 3$ presentaban invasión vascular y uno de ellos tenía asociado un componente de adenocarcinoma.

En 11 (68,7\%) pacientes había otras lesiones colónicas sincrónicas: 10 pólipos (ocho adenomatosos y dos hiperplásicos), una colitis ulcerosa, una colitis crónica inespecífica y un carcinoma in situ más un mucocele apendicular.

Tras el tratamiento quirúrgico, cuatro pacientes con un TNER G3 recibieron tratamiento adyuvante, tres con quimioterapia (asociando radioterapia en dos de
Tabla 1. Clasificación TNM, estadio y grado en el momento del diagnóstico en los 16 tumores neuroendocrinos rectales

\begin{tabular}{|c|c|c|c|c|c|c|c|c|c|}
\hline$T$ & n (\%) & $N$ & $n(\%)$ & M & $n(\%)$ & Estadio & n (\%) & Grado & n (\%) \\
\hline T1a & $10(62)$ & $\mathrm{Nx}$ & - & MO & - & I & $11(69)$ & G1 & $11(69)$ \\
\hline $\mathrm{T} 1 \mathrm{~b}$ & $2(12)$ & NO & - & M1 & $3(19)$ & Ila & - & G2 & - \\
\hline T2 & $1(6)$ & N1 & $5(31)$ & & & Ilb & - & G3 & $5(31)$ \\
\hline T3 & $1(6)$ & & & & & IIla & - & & \\
\hline \multirow[t]{2}{*}{$\mathrm{T} 4$} & $2(12)$ & & & & & IIlb & $2(12)$ & & \\
\hline & & & & & & IV & $3(19)$ & & \\
\hline
\end{tabular}

ellos) y uno (con síndrome carcinoide) con análogos de la somatostatina (ASS).

La media de seguimiento fue de 26 meses, con una mediana de 12 (rango: 0,5-109). Ninguno de los pacientes estudiados presentó recidiva local. Un TNER de grado G1 con márgenes positivos tras la resección endoscópica presentó valores elevados de $\mathrm{CgA}$, por lo que se realizaron controles con SPECT, endoscopia y USE, que resultaron normales, y se le dio de alta tras 5 años de revisiones periódicas. Durante el seguimiento fallecieron $3(18,7 \%)$ pacientes, todos ellos con TNER de grado G3 metastásico, y la supervivencia media fue de 8,3 meses.

\section{Discusión}

Los TNER han pasado de considerarse un tipo poco frecuente de neoplasia rectal a ser un hallazgo cada vez más habitual en la práctica clínica ${ }^{19}$. La amplia difusión de las pruebas endoscópicas, las campañas de cribado del carcinoma colorrectal, la generalización de los estudios histológicos empleando marcadores neuroendocrinos y la mejor recogida de datos en los registros hospitalarios son los factores que explicarían esta mayor incidencia ${ }^{7,9,10,20,21}$. En nuestro trabajo, el $81 \%$ de los casos se diagnosticaron en los últimos años del estudio, lo que también se atribuye a los factores expuestos previamente.

La localización anal de los TNE es muy rara y, como en el único caso de nuestra serie, suelen ser neoplasias agresivas ${ }^{22}$. En la revisión realizada por el Instituto Nacional del Cáncer, Epidemiología y Resultados Finales de los EE.UU., que abarca desde el año 1950 hasta el año 2007, de los 31,644 TNEGEP registrados solo el $0.2 \%$ se localizaban en la región anal ${ }^{7}$, mientras que en el RGETNE no se encuentra ningún tumor localizado en el ano ${ }^{13}$.

En el momento del diagnóstico, la mayoría de los carcinoides rectales (75-96\%) están localizados, lo 
que coincide con nuestra serie (69\%). La existencia de metástasis varía según el tamaño y el grado de agresividad del tumos ${ }^{10,12,15,23}$; en nuestra revisión hemos observado que todos los tumores con enfermedad diseminada en el momento de su diagnóstico eran G3.

La media de edad de los pacientes con TNER en la mayoría de las series, así como en la nuestra, se halla entre la quinta y la sexta décadas de la vida $7,8,19,21,24-27$. El sexo masculino es el más afectado por los TNER ${ }^{8}$, lo cual es muy evidente en nuestra serie, con un $75 \%$ de varones, aunque esta diferencia no es tan marcada en otros trabajos ${ }^{13,21,27,28} \mathrm{e}$ incluso en algunos predominan en las mujeres ${ }^{24,26}$.

Los aspectos clínicos fueron similares a los publicados en la literatura, ya que la mayoría de los TNER suelen presentarse en pacientes asintomáticos. Cuando existe clínica, suelen predominar los síntomas locales, como rectorragia, dolor anorrectal, pérdida de peso inexplicable o alteración del ritmo intestinal. La presencia de un síndrome carcinoide típico es rara y suele ocurrir en casos con enfermedad metastási$\mathrm{ca}^{23}$, como sucede en los $2(12,5 \%)$ casos de nuestra serie.

La principal prueba diagnóstica en los TNER es la endoscopia baja, pues la mayoría de estos tumores se localizan entre los 5 y $10 \mathrm{~cm}$ desde el margen anal ${ }^{3,27}$. Su aspecto típico es una lesión submucosa, redondeada, de consistencia blanda, recubierta de una mucosa de color normal o amarillenta. La presencia de ulceración, depresión central o hiperemia es atípica y se asocia con invasión parietal y linfática ${ }^{29,30}$. La colonoscopia debe ser completa para poder descartar otras lesiones sincrónicas, como el adenocarcinoma, cuya asociación es frecuente ${ }^{6,12,23}$ (un $12.5 \%$ en nuestra revisión).

Otra prueba diagnóstica es la determinación de 5-HIAA, aunque es poco útil ya que muy pocos TNER producen serotonina. Las concentraciones séricas de $\mathrm{CgA}$ suelen ser normales, excepto cuando existe enfermedad metastásica, y se recomienda su determinación como marcador tumoral en el seguimiento de los pacientes con mal pronóstico ${ }^{19}$. Otras determinaciones que pueden alterarse en estos tumores son el antígeno carcinoembrionario, el antígeno específico de próstata, la B-hormona gonadotropina coriónica, el polipéptido pancreático y el péptido glucagon-like ${ }^{3,23}$.

La USE y la RM también son métodos diagnósticos de gran utilidad para determinar la invasión de la pared rectal y la existencia de adenopatías regionales $^{29}$. Su empleo puede ser útil para determinar si se va a realizar un tratamiento endoscópico o una resección quirúrgica en aquellos casos endoscópicamente atípicos, en lesiones $>1 \mathrm{~cm}$, o si en el estudio histológico existe invasión de márgenes o datos de mal pronóstico (infiltración de la muscular propia, invasión linfovascular, índice mitótico >2/10 campo de gran aumento (HPF) o Ki-67 > 2\%) $)^{3,10,19,31,32}$.

Para la detección de metástasis a distancia, las pruebas de elección son la TC toracoabdominal y la RM hepática ${ }^{23}$.

La GASS o la SPECT están indicadas en tumores > $2 \mathrm{~cm}$ para la valoración de la diseminación metastásica; también son útiles para la selección y la monitorización de los tratamientos con ASS o radionúclidos. La PET estaría indicada en los tumores indiferenciados, en los que la GASS suele ser negativa ${ }^{3,23}$.

El único tratamiento curativo de los TNER es su resección completa ${ }^{23}$. Las opciones terapéuticas varían según su tamaño, aspecto endoscópico y factores de riesgo $0^{10,24,25,29}$. Aproximadamente un $78 \%$ de Ios TNER se tratan por vía endoscópica, un 10\% con cirugía endoanal y un $12 \%$ con cirugía radical ${ }^{21}$; cifras similares a las que observamos en nuestra serie.

En los tumores $\leq 1 \mathrm{~cm}$ localizados en la mucosa/ submucosa puede realizarse una polipectomía simple ${ }^{15,33}$ o una resección endoscópica de la mucosa con cabezal de aspiración o con ligadura elástica ${ }^{3,28,34}$. Para obtener una mayor profundidad en la resección, algunos autores proponen una disección endoscópica submucosa ${ }^{26,35}$. Las técnicas de resección endoscópica avanzada parecen logran una mayor tasa de márgenes negativos, sin que aumenten de manera significativa las complicaciones ${ }^{10,36,37}$, aunque ciertos autores no encuentran diferencias significativas y consideran que la técnica endoscópica debe elegirse teniendo en cuenta factores como el coste-efectividad y la experiencia de cada centro ${ }^{38}$.

La existencia de márgenes afectados 0 indeterminados tras la resección endoscópica oscila entre un 16 y un $83 \%{ }^{33,39-41}$. En nuestra serie se observó un $43.7 \%$ de márgenes indeterminados y un $37.5 \%$ de márgenes afectados. En los tumores diferenciados no parece tener importancia pronóstica la afectación de los bordes de resección, aunque obliga a realizar un control con USE y un seguimiento más estricto del paciente, a diferencia de los casos de mal pronóstico, en los que la existencia de márgenes positivos obliga a una nueva resección más agresiva ${ }^{3,27,40}$.

Los tumores $>2 \mathrm{~cm}$ que invaden la capa muscular propia o con criterios histológicos de agresividad deben tratarse con cirugía radical, de modo similar al 
adenocarcinoma $3,5,14,19,30,42$. En los TNER con diseminación a distancia, esta cirugía no parece aportar una mayor supervivencia, pero puede mejorar la calidad de vida y evitar complicaciones $3,23,32,43$.

El tratamiento de los casos intermedios $(1-2 \mathrm{~cm})$ es un tema más debatido ${ }^{4}$, porque si se localizan en la mucosa-submucosa y no presentan factores de riesgo pueden tratarse con microcirugía transanal o con resección endoscópica avanzada ${ }^{3,27}$, y si son $>1,5 \mathrm{~cm}$ o tienen factores de riesgo debe realizarse una cirugía radical10.

El tratamiento adyuvante con quimioterapia sistémica está indicado en los tumores pobremente diferenciados $^{44}$. Los ASS pueden controlar los síntomas del síndrome carcinoide y, al igual que el interferón, su empleo como agentes antitumorales tiene una evidencia limitada. Asimismo, en los tumores metastásicos inoperables con GASS positiva puede emplearse radioterapia con ASS marcados con radionúclidos 3,10,19,45. En nuestra serie, todos los pacientes con TNER G3 recibieron quimioterapia, y en dos de ellos se asoció radioterapia.

Existen pocos estudios específicos sobre el manejo de la enfermedad metastásica hepática en los TNER. La resección hepática, que abarca desde una metastasectomía hasta un trasplante hepático, puede realizarse con un fin curativo, para el control de los síntomas o para la reducción de la masa tumoral. Como tratamientos alternativos o asociados a la resección hepática se emplean la ablación con radiofrecuencia y la embolización o quimioembolización transarterial ${ }^{3}$.

Los TNER $<1 \mathrm{~cm}$ extirpados por completo y $\sin$ factores de riesgo no precisan seguimiento oncológico, aunque tras una resección endoscópica es recomendable la realización de una rectoscopia cada 6 meses durante 2 años. En los tumores con riesgo de recidiva local o de metástasis se recomienda realizar controles periódicos durante 5 a 10 años con endoscopia, RM, USE, TC y determinaciones de $\mathrm{Cg}^{19,23,46,47}$.

La supervivencia a los 5 años de los pacientes con TNER bien o moderadamente diferenciados varía en función de su estadio: 92-100\% para el I, 80-88\% para el II; $51-59 \%$ para el III y $0-15 \%$ para el IV $8,11,25,33,48,49$. Los tumores con alto grado de agresividad tienen un mal pronóstico, incluso aquellos que están localizados, con una supervivencia media de 13 meses $^{43}$. En nuestra revisión, en los pacientes con TNER de grado G3 hallamos una supervivencia menor de 8.3 meses.

\section{Conclusiones}

Los TNER son cada vez más frecuentes en la práctica clínica, ya que su incidencia está aumentando debido a la mayor difusión de los estudios endoscópicos, a las campañas de cribado del carcinoma colorrectal, a la generalización de los estudios histológicos con marcadores neuroendocrinos específicos, a la mejor recogida de datos en los registros hospitalarios y a la mayor concienciación de la existencia de estos tumores. Su prevalencia también está aumentando por tratarse de pacientes con una supervivencia más prolongada. Debemos tener presente la necesidad de un equipo multidisciplinario que sea el encargado de su manejo, en especial en los casos avanzados, según las guías consensuadas por las diversas sociedades científicas $4,6,21,23,46,47,50,51$, para evitar la realización de costosas pruebas diagnósticas poco eficaces, conseguir la estandarización de su tratamiento y realizar el seguimiento adecuado para cada paciente.

\section{Financiación}

Este trabajo no ha sido financiado por ninguna organización pública ni privada.

\section{Conflicto de intereses}

Los autores no presentan conflicto de intereses.

\section{Bibliografía}

1. Lubarsch O. Uber den primaren Krebs des lleum nebst Bemerkungen uber das gleichzeitige Vorkommen von Krebs und Tuberkulose. Virchows Arch. 1888;3:280-317.

2. Oberndorfer S. Karzinoid Tumore des Dunndarms. Frankf Z Pathol. 1907;1:426-30.

3. Mandair D, Caplin M. Colonic and rectal NET's. Best Pract Res Clin Gastroenterol. 2012;26:775-89.

4. Ramage J, Ahmed A, Ardill J, Bax N, Breen J, Caplin M, et al. Guidelines for the management of gastroentero-pancreatic neuroendocrine (including carcinoid) tumours (NETs). Gut. 2012;61:6-32.

5. Pasieka J. Carcinoids tumors. Surg Clin N Am. 2009;89:1123-37.

6. Kocha W, Maroun J, Kennecke H, Law C, Metrakos P, Ouellet J, et al. Consensus recommendations for the diagnosis and management of weII-differentiated gastroenterohepatic neuroendocrine tumours: a revised statement from a Canadian National Expert Group. Curr Oncol. 2010; 17:49-63.

7. Lawrence B, Gustafsson B, Chan A, Svejda B, Kidd M, Modlin I. The epidemiology of gastroenteropancreatic neuroendocrine tumors. Endocrinol Metab Clin N Am. 2011;40:1-18.

8. Yao JC, Hassan M, Phan A, Dagohoy C, Leary C, Mares J, et al. One hundred years after "carcinoid": epidemiology of and prognostic factors for neuroendocrine tumors in 35,825 cases in the United States. J Clin Oncol. 2008;26:3063-72.

9. Jetmore A, Ray J, Gathright J, McMullen K, Hicks T, Timmeke A. Rectal carcinoids: the most frequent carcinoid tumor. Dis Colon Rectum. 1992;35:717-25.

10. De Mestier L, Brixi H, Gincul R, Ponchon T, Cadiot G. Updating the management of patients with rectal neuroendocrine tumors. Endoscopy. 2013;45:1039-46.

11. Modlin I, Lye K, Kidd M. A 5-decade analysis of 13,715 carcinoid tumors. Cancer. 2003;97:934-59. 


\section{B. De Andrés-Asenjo, et al.: Tumor neuroendocrino ano-rectal}

12. Modlin I, Kidd M, Latich I, Zikusoka M, Shapiro M. Current status of gastrointestinal carcinoids. Gastroenterology. 2005;128:1717-51.

13. García-Carbonero R, Capdevila J, Crespo-Herrero G, Díaz-Pérez J, Martínez del Prado, Alonso Orduña V, et al. Incidence, patterns of care and prognostic factors for outcome of gastroenteropancreatic neuroendocrine tumors (GEP-NETs): results from the National Cancer Registry of Spain (RGETNE). Ann Oncol. 2010;21:1794-803.

14. Partelli S, Maurizi A, Tamburrino D, Baldoni A, Polenta V, Crippa S, et al. A review on surgery of gastro-entero-pancreatic neuroendocrine tumors. Eur J Endocrinol. 2014;171:153-62.

15. Mani S, Modlin I, Ballantyne G, Ahlman H, West B. Carcinoids of the rectum. J Am Coll Surg. 1994;179:231-48.

16. Jann H, Roll S, Couvelard A, Hentic O, Pavel M, Müller-Nordhorn J, et al. Neuroendocrine tumors of midgut and hindgut origin: tumor-node-metastasis classification determines clinical outcome. Cancer. 2011;117: 3332-41.

17. UICC (International Union Against Cancer). TNM Classification of malignant tumours. $7^{\text {th }}$ ed. Chichester, West Sussex, UK; Hoboken, NJ: Wiley-Blackwell; 2009.

18. Bosman F, Carneiro F, Hruban R, Theise N, editores. WHO Classification of tumours of the digestive system. Lyon, France: IARC; 2010.

19. Errasti J, Espín E, Reina A. Neoplasias de recto poco frecuentes. Revisión de conjunto. Cir Esp. 2014;92:579-88.

20. Fraenkel M, Kim M, Faggiano A, Herder W, Valk G. Incidence of gastro-enteropancreatic neuroendocrine tumours: a systematic review of the literature. Endocr Relat Cancer. 2014;21:153-63.

21. McDermott F, Heeney A, Courtney D, Mohan H, Winter D. Rectal carcinoids: a systematic review. Surg Endosc. 2014;28:2020-6.

22. Sebastián Ayala $R$, Concha Sierralta $M$, Domínguez Vera $A$ Canelo Ruiz P. Tumor carcinoide de ano, presentación de un caso y revisión de la literatura. Revista de la Facultad de Medicina Humana de la Universidad Ricardo Palma. 2013;1:42-5.

23. Caplin M, Sundin A, Nillson O, Baum P, Klose K, Kelestimur F, et al ENETS consensus guidelines for the management of patients with digestive neuroendocrine neoplasms: colorectal neuroendocrine neoplasms. Neuroendocrinology. 2012;95:88-97.

24. Fahy B, Tang L, Klimstra D, Wong W, Guillem J, Paty P, et al. Carcinoid of the rectum risk stratification (CaRRs): a strategy for preoperative outcome assessment. Ann Surg Oncol. 2007;14:1735-43.

25. Shields C, Tiret E, Winter D, International Rectal Carcinoid Study Group. Carcinoid tumors of the rectum: a multi-institutional international collaboration. Ann Surg. 2010;252:750-5.

26. Ono A, Fujii T, Saito Y, Matsuda T, Lee DT, Gotoda T, et al. Endoscopic submucosal resection of rectal carcinoid tumors with a ligation device. Gastrointest Endosc. 2003;57:583-7

27. Kumar A, Sidani S, Kolli K, Stahl T, Ayscue J, Fitzgerald J, et al. Transanal endoscopic microsurgery for rectal carcinoids: the largest reported United States experience. Colorectal Dis. 2012;14:562-6.

28. Ono A, Fujii T, Saito Y, Matsuda T, Lee D, Gotoda T, et al. Endoscopic submucosal resection of rectal carcinoid tumors with a ligation device. Gastrointest Endosc. 2003;57:583-7.

29. Zhou X, Xie H, Xie L, Li J, Fu W. Factors associated with lymph node metastasis in radically resected rectal carcinoids: a systematic review and meta-analysis. J Gastrointest Surg. 2013;17:1689-97.

30. Attili F, Capurso G, Vanella G, Fuccio L, Delle Fave G, Costamanga G, et al. Diagnostic and therapeutic role of endoscopy in gastroenteropancreatic neuroendocrine neoplasms. Dig Liver Dis. 2014:46:9-17.

31. Fujishima H, Misawa T, Maruoka A, Yoshinaga M, Chijiiwa Y, Nawata H. Rectal carcinoid tumor: endoscopic ultrasonographic detection and endoscopic removal. Eur J Radiol. 1993;16:198-200.

32. Koura A, Giacco G, Curley S, Skibber J, Feig B, Ellis L. Carcinoid tumors of the rectum: effect of size, histopathology, and surgical treatment on metastasis free survival. Cancer. 1997;79:1294-8.

33. Kim G, Kim K, Hong S, Yu E, Yang D, Jung K, et al. Clinical outcomes of rectal neuroendocrine tumors $\leq 10 \mathrm{~mm}$ following endoscopic resection. Endoscopy. 2013;45:1018-23.

34. Imada-Shirakata Y, Sakai M, Kajiyama T, Kin T, Inoue K, Torii A, et al Endoscopic resection of rectal carcinoid tumors using aspiration lumpectomy. Endoscopy. 1997;29:34-8.

35. Fujishiro M, Yahagi N, Nakamura M, Kakushima N, Kodashima S, Ono S, et al. Successful outcomes of a novel endoscopic treatment for Gl tumors: endoscopic submucosal dissection with a mixture of high-molecular-weight hyaluronic acid, glycerin, and sugar. Gastrointest Endosc. 2006;63:243-9.

36. Zhong D, Shao L, Cai J. Endoscopic mucosal resection vs endoscopic submucosal dissection for rectal carcinoid tumours: a systematic review and meta-analysis. Colorectal Dis. 2012;15:283-91.

37. Zhou X, Xie H, Xie L, Li J, Cao W, Fu W. Endoscopic resection therapies for rectal neuroendocrine tumors: a systematic review and meta-analysis. J Gastroenterol Hepatol. 2014;29:259-68.

38. Onozato Y, Kakizaki S, lizuka H, Sohara N, Mori M, Itoh H. Endoscopic treatment of rectal carcinoid tumors. Dis Colon Rectum. 2010:53:169-76.

39. Kobayashi K, Katsumata T, Yoshizawa S, Sada M, Igarashi M, Saigenji K, et al. Indications of endoscopic polypectomy for rectal carcinoid tumors and clinical usefulness of endoscopic ultrasonography. Dis Colon Rectum. 2005;48:285-91.

40. Kwaan M, Goldberg J, Bleday R. Rectal carcinoid tumors: review of results after endoscopic and surgical therapy. Arch Surg. 2008;143:471-5.

41. Park C, Cheon J, Kim J, Shin J, Jang B, Shin S, et al. Criteria for decision making after endoscopic resection of well-differentiated rectal carcinoids with regard to potential lymphatic spread. Endoscopy. 2011; 43:790-5.

42. Larrad A. Síndrome carcinoide. Cir Esp. 1997;62:224-33.

43. Smith J, Reidy D, Goodman K, Shia J, Nash G. A retrospective review of 126 high-grade neuroendocrine carcinomas of the colon and rectum. Ann Surg Oncol. 2014;21:2956-62.

44. Sorbye H, Strosberg J, Baudin E, Klimstra D, Yao J. Gastroenteropancreatic high-grade neuroendocrine carcinoma. Cancer. 2014;120:2814-23.

45. Castellano D, Bajetta E, Panneerselvam A, Stephen S, Kocha W, O'Drisio T, et al. Everolimus plus octreotide long-acting repeatable in patients with colorectal neuroendocrine tumors: a subgroup analysis of the phase III RADIANT-2 study. Oncologist. 2013:18:46-53.

46. Kulke M, Shah M, Benson A, Bergsland E, Berlin J, Blaszkowky L, et al. Neuroendocrine tumors. Version I.J Natl Compr Canc Netw. 2015 Jan; 13(1):78-108.

47. Escudero MP, Acha J, Alonso V et al. TNE intestinales. Guía Práctica de Diagnóstico, Tratamiento y Seguimiento de Tumores Neuroendocrinos, Versión 3. Barcelona: EdikaMed; 2016. p. 99-120.

48. Chagpar R, Chian Y, Xing Y, Cormier J, Feig B, Rashid A, et al. Neuroendocrine tumors of the colon and rectum: prognostic relevance and comparative performance of current staging systems. Ann Surg Oncol. 2013:20:1170-8.

49. Kim M, Hur H, Min B, Baik S, Lee K, Kim N. Clinical outcomes for rectal carcinoid tumors according to a new (AJCC $7^{\text {th }}$ edition) TNM staging system: a single institutional analysis of 122 patients. J Surg Oncol. 2013; 107:835-41

50. Kunz P, Reidy-Lagunes D, Anthony L, Bertino E, Brendtro K, Chan J, et al. Consensus guidelines for the management and treatment of neuroendocrine tumors. Pancreas. 2013;42:557-77.

51. Öberg K, Knigge U, Kwekkeboom D, Perren A. Neuroendocrine gastro-entero-pancreatic tumors: ESMO clinical practice guidelines for diagnosis, treatment and follow up. Ann Oncol. 2012;23:124-30. 